\title{
On Optimizing Fractional Lower Order Covariance based Synchronization Method for Random Communication Systems
}

\author{
Areeb Ahmed and Ferit Acar Savaci \\ Department of Electrical and Electronic Engineering \\ ${ }^{1}$ Izmir Institute of Technology \\ Izmir, Turkey \\ areebahmed@iyte.com.tr and acarsavaci@iyte.edu.tr
}

\begin{abstract}
Previously, the concept of Pilot Assisted Synchronization (PAS) and Fractional Lower Order Covariance (FLOC) has been incorporated together to synchronize alphastable noise based communication system which gave birth to Synchronized Random Communication Systems (SRCS). In this paper, an optimization criterion, i.e. FLOC Margin, has been proposed to improve the efficiency of the Fractional Lower Order Covariance based Correlators (FLOCCs) at the receiver side, hence, resulting in enhanced Bit Error Rate (BER) performance of SRCS. Since, the characteristic exponent and impulsiveness parameter are mainly responsible in generating and shaping up the required pilot sequence, therefore, the effects of these parameters on BER performance has also been observed by fluctuating them in their respective ranges. It has been shown that the proposed optimization criterion increases the BER efficiency of SRCS and also reveals the specific range of the characteristic exponent and impulsiveness parameter which can be exploited to achieve the optimum performance.
\end{abstract}

Keywords-Synchronization; Optimization; Fractional lower order covariance; Alpha-stable noise; Random Communication system; Modified extreme value method

\section{INTRODUCTION}

Noise, either manmade or natural, had always been considered as useless or harmful in any type of communication system until the idea to establish secure communications by exploiting the underlying spread spectrum characteristics of noise started in 1950's [1]. The concept gained further attention when Stochastic or Random noise, i.e. Auto Regressive Moving Average (ARMA) process, was first utilized to perform covert communications which originated Stochastic Communication Systems [2]. Later on, the impulsiveness and skewness parameter of alpha $(\alpha)$-stable noise were used to propose symmetric and skewed $\alpha$-stable noise based communication systems, respectively $[3,4]$. Since, second order statistics of $\alpha$-stable distribution does not exist and currently no method, algorithm or attack is available to crack real time $\alpha$-stable noise signals, therefore, $\alpha$-stable noise based communication systems are considered more secure in comparison to other noise based communication systems. Consequently, studies were also conducted to extend the scope of binary, i.e. 2-ary, to $\mathrm{M}$-ary $\alpha$-stable noise based communication systems [5]. Additionally, $\alpha$-stable noise based communication systems have shown efficient performance in Additive White Gaussian Noise (AWGN) and Multipath fading channels as well [6]. Similarly, a covert communication system based on normal distribution, i.e. a special case of $\alpha$-stable distribution, has also been proposed [7]. Recently, the most optimized model of $\alpha$-stable noise based communication system, i.e. Random Communication System (RCS), has been introduced [8]. However, all the $\alpha$-stable noise based communication systems discussed above used to assume perfect synchronization where no method was actually introduced to establish synchronization in RCSs and it has also been shown in [9] that imperfect synchronization has drastic effects on the performance of RCSs. Therefore, Synchronized Random Communication System (SRCS) consisting of Fractional Lower Order Covariance based Correlators (FLOCCs), Threshold Detectors (TDs) and Modified Extreme Value Method (MEVM) estimator was proposed in [10]. However, further investigation is required to analyze the effects of various parameters on SRCS's BER performance by varying the associated parameters in their defined ranges. Similarly, a criterion is necessary to prescribe the best range of values for the associated parameters involve in the structure of SRCS.

Therefore, in this paper, an optimization criteria, i.e. FLOC Margin, has been proposed to increase the efficiency of SRCS. The effects of maneuvering the characteristic exponent and impulsiveness parameter on SRCS's BER performance have also been analyzed. Moreover, specific range of the characteristic exponent and impulsiveness parameter, needed to achieve the optimal BER performance, has also been advised. In the following Section-II, SRCS has been reviewed. The proposed optimization criterion has been given in Section-III. The performance of SRCS in accordance to the proposed criterion has been shown in Section-IV followed by a conclusion in Section-V.

\section{SYNCHRONIZED RANDOM COMMUNICATION SYSTEM}

The SRCS, as proposed in [10], has been shown in Fig. 1. It consists of $\alpha$-stable noise shift keying modulator ( $\alpha$-SNSKM) and Transmitter Synchronization Block (TSB) on the transmitter side while MEVM estimator and Receiver Synchronization Block (RSB) on the receiver side. The AWGN has been used as a channel noise to contaminate the transmitted $\alpha$-stable noise signals. The transmitter, receiver and the sub 

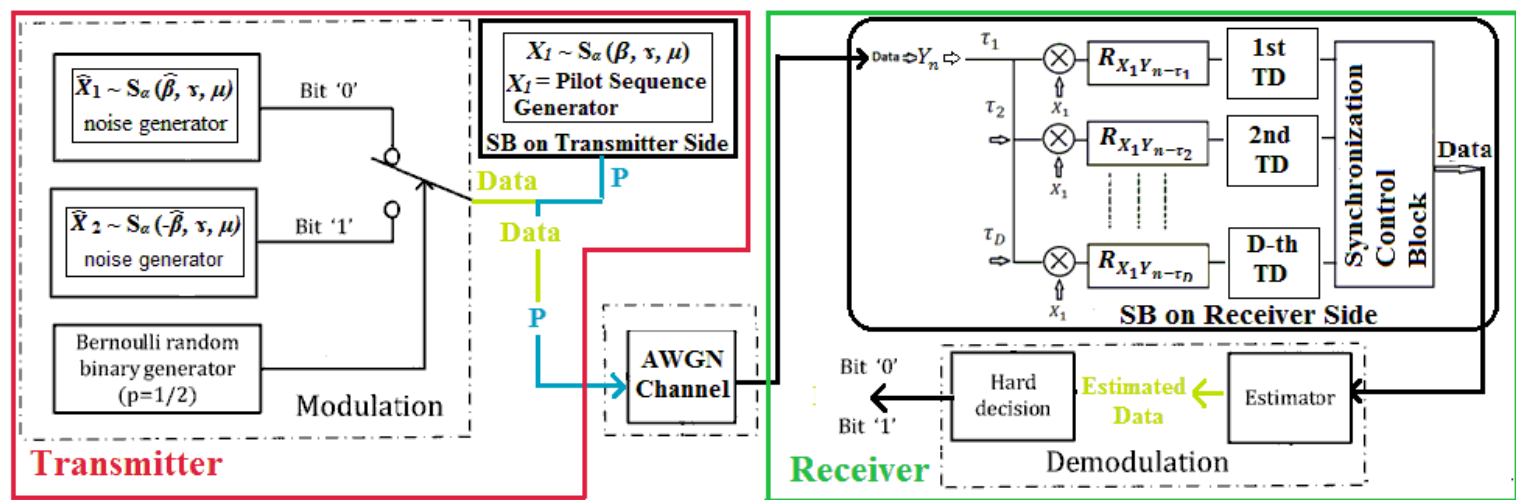

Fig. 1: System model of Synchronized $\alpha$-stable noise based Random Communication system (SRCS)

blocks have been explained in the following subsections after a brief introduction of $\alpha$-stable distribution.

\section{A. $\alpha$-Stable Distribution}

$\alpha$-stable distribution or noise ' $\mathrm{X}$ ' is represented as $\mathrm{X} \sim \mathrm{S}_{\alpha}$ $(\beta, \gamma, \mu)$ where $\alpha(0<\alpha \leq 2)$ is the characteristic exponent, $\beta(-1 \leq \beta \leq 1)$ is the skewness parameter, $\gamma(\gamma \geq 0)$ is the dispersion parameter and $\mu \in R$ is the location parameter [11]. The second or higher order moments of $\alpha$-stable distribution does not exist for $\alpha<2$. The $\alpha$-stable stable distribution can be generated as a pilot sequence or as a channel noise by the method given in [12].

\section{B. Transmitter side}

The transmitter consists of $\alpha$-SNSKM and TSB. The $\alpha$-stable noise used as a pilot sequence in TSB or as a message sequence in $\alpha$-SNSKM are obtained from separate $\alpha$-stable distributions represented as $X_{1} \sim \mathrm{S}_{\alpha}(\beta, \gamma, \mu), \hat{X}_{1} \sim \mathrm{S}_{\alpha}(-\hat{\beta}, \gamma, \mu)$ and $\hat{X}_{2} \sim \mathrm{S}_{\alpha}(\hat{\beta}, \gamma, \mu)$ where $\hat{\beta} \neq \beta$. The TSB and $\alpha$-SNSKM are defined below as

$\alpha$-SNSKM: The binary message ' 0 ' and ' 1 ' are transmitted by random noise $\hat{X}_{1}$, i.e. $\hat{X}_{1} \sim \mathrm{S}_{\alpha}(-\hat{\beta}, \gamma, \mu)$, and $\hat{X}_{2}$, i.e. $\hat{X}_{2} \sim \mathrm{S}_{\alpha}$ $(\hat{\beta}, \gamma, \mu)$, respectively, by utilizing the antipodal characteristics of skewed $\alpha$-stable distributions which can be obtained by bending the distribution either to the right, i.e. $\hat{\beta}=1$, or to the left, i.e. $\hat{\beta}=-1$.

TSB: The pilot sequence and its related aspects, e.g. the cut off threshold, pre known to the transmitter and the intended receiver are generated by TSB. Pilot sequence $P_{l}$ consisting of ' $m$ ' repeated pilot symbol $X_{1}$ is described as

$$
P_{1}=\left[X_{1}, X_{1}, \ldots \ldots, X_{1}\right]_{1 \times m N} \mid X_{1}=\left[x_{1} x_{2} \ldots \ldots . x_{N}\right]_{1 \times N}
$$

and each pilot symbol consists of ' $N$ ' $\alpha$-stable noise samples. Every $n$-th pilot symbol ' $X_{n}$ ' is created from the $\alpha$-stable noise samples of $X_{1}$ by the relation given below as

$$
X_{n}=\left[x_{n} \ldots \ldots x_{N-1+n}\right]_{1 \times N} \mid n=1,2 \ldots(m-1) N+1
$$

and

$$
x_{n}=x_{u N+n} \quad \mid u=0,1,2 \ldots m-1
$$

Therefore $P_{n}$ is represented as

$$
P_{n}=\left[X_{n}, X_{n} \ldots \ldots X_{n}\right]_{1 \times m N}
$$

The pilot symbols ' $X_{n}$ ' are then made noisy as

$$
X_{n}=X_{n}+G \quad \text { and } \quad G \sim S_{\alpha=2}(\beta, \gamma, \mu)
$$

And the FLOC vector ' $R$ ' based on the members FLOCs $R_{X_{1} X_{k}}$ are obtained as

$$
R=\left[\begin{array}{lllllll}
R_{X_{1} X_{2}} & R_{X_{1} X_{3}} \ldots \ldots & R_{X_{1} X_{k}} & \ldots & \cdots & \cdots & R_{X_{1} X_{N+1}}
\end{array}\right]_{1 \times N}
$$

where the FLOC method, i.e. $R_{X_{1} X_{k}}$, has been defined as

$R_{X_{1} X_{k}}=\frac{\sum_{n=N_{1}+1}^{N_{2}}\left|x_{1}[n]\right|^{a} \cdot\left|x_{k}[n]\right|^{b} \cdot \operatorname{sign}\left(x_{1}[n] \cdot x_{k}[n]\right)}{N_{2}-N_{1}} \mid k=2 \ldots N+1(7)$

and $N_{l}=0, N_{2}=N$ and the fractional powers are $a=b=\frac{\alpha}{2}$.

The cut-off threshold $L_{t h}$ has been pre-computed as

$$
L_{t h}=\frac{\|R\|_{\infty}+\left\|R_{\text {red }}\right\|_{\infty}}{2}
$$

where $\|R\|_{\infty}=\max _{1 \leq i \leq N}\left|r_{i}\right|=\left|r_{L}\right|$ and $R_{\text {red }}$ is the reduced vector obtained by deleting $r_{L}$ from $R$.

\section{Receiver side}

The receiver consists of RSB and MEVM estimator. RSB: There are total ' $D$ ' FLOCCs and TDs and a single SCB, as shown in Fig. 1. The received pilot sequence ' $Y_{n}$ ' by RSB is

$$
Y_{n}=P_{n}+N_{\text {channel }}
$$

where $N_{\text {channel }}$ is the actual channel noise, i.e. $G \neq N_{\text {channel }}$, defined below as

$$
N_{\text {channel }} \sim S_{\alpha=2}\left(0, \gamma_{\text {Nchannel }}=1,0\right)
$$


MEVM estimator: The receiver extracts hidden binary message by estimating $\hat{\beta}$ using Maximum Extreme Value Method in $[10,13]$ and a hard decision is taken on $\hat{\beta}$ to decode the binary message as ' 1 ', i.e. $\hat{\beta} \geq 0$, or ' 0 ', i.e. $\hat{\beta}<0$.

\section{OPTIMIZATION CRITERIA AND PARAMETERS}

It is known that $L_{t h}$ is pre-computed in SRCS to permit the intended receiver to initiate the threshold detection process and facilitate in taking the hard decision. Since, $L_{t h}$ is decided by the maximum and minimum FLOC values for $R_{X_{1} X_{k}}$, i.e. $\|R\|_{\infty}$ and $\left\|R_{\text {red }}\right\|_{\infty}$, respectively, therefore, it is necessary to study the effects of maneuvering the difference between $\|R\|_{\infty}$ and $\left\|R_{\text {red }}\right\|_{\infty}$ by fluctuating $\alpha$ and $\beta$ in their respective ranges. The idea will help in improving the detection process of the intended receiver by identifying the requisite pilot symbol $X_{1}$ much easily among all other un-requisite pilot symbols $X_{k}$. Similarly, the best values of $\alpha$ and $\beta$ can also be determined which can be used to increase the difference between $\|R\|_{\infty}$ and $\left\|R_{\text {red }}\right\|_{\infty}$, hence, resulting in optimized SRCS's BER performance.

\section{A. FLOC Margin}

The proposed optimization criterion, i.e. FLOC Margin, denoted by ' $\mathbf{M}$ ', is defined as

$$
\mathbf{M}=\|R\|_{\infty}-\left\|R_{\text {red }}\right\|_{\infty}
$$

The SRCS would be considered as optimized as big the $\mathbf{M}$, i.e. as bigger difference between $\|R\|_{\infty}$ and $\| R$ red $\|_{\infty}$, hence, resulting in better BER performance. In contrast, $\mathbf{M}$ close to zero, i.e. as the difference between $\|R\|_{\infty}$ and $\left\|R_{\text {red }}\right\|_{\infty}$

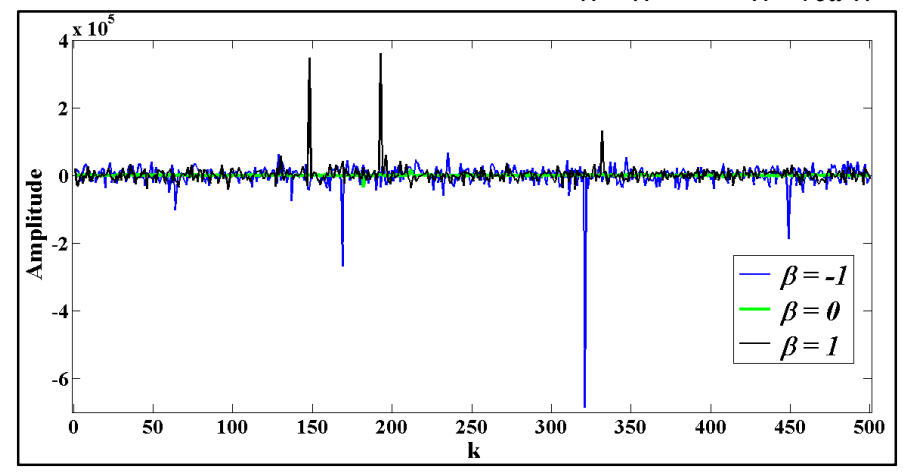

Fig. 2: Pilot Sequences taken from $\mathrm{S}_{\boldsymbol{\alpha}=\mathbf{0 . 5}}(\beta, \gamma=1, \mu=0)$

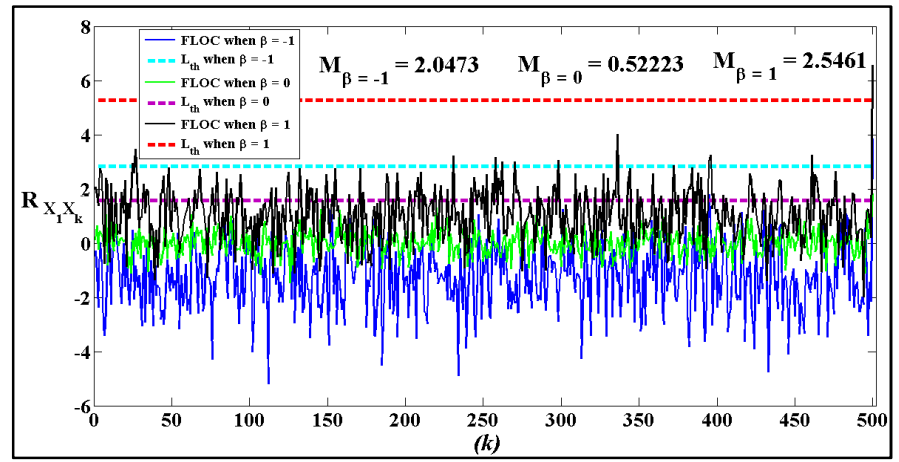

Fig. 3: FLOCs of $\mathbf{X}_{\mathbf{1}} \mathbf{X}_{\mathbf{k}}$ for Pilot Sequences taken from $\mathrm{S}_{\boldsymbol{\alpha}=\mathbf{0 . 5}}(\beta$, $\boldsymbol{\gamma}=1, \mu=0$ ) with corresponding threshold margins ' $\mathrm{M}$ ' becomes smaller, results in worst BER performance of SRCS.

\section{B. Optimized Parameter values}

According to the proposed criterion of FLOC Margin, the values of $\alpha$ and $\beta$ which correspond to greater $\mathbf{M}$ are expected to give optimized BER performance, hence, referred as Optimized Parameter Values, i.e. OPV, of SRCS. Both $\mathbf{M}$ and OPV can be used to obtain optimum BER performance in SRCS, i.e. RCS synchronized by $\alpha$-stable pilot sequence, and also for all other RCSs which are synchronized by any other pilot sequence.

The performance of the SRCS has been evaluated in accordance to the proposed criterion of $\mathbf{M}$ and $\mathbf{O P V}$ by BER vs. Mixed Signal to Noise Ratio $(M S N R)$ where $M S N R$ is given in $[13]$ as

$$
M S N R_{d B}=10 \log \frac{\gamma}{\gamma_{N_{\text {channel }}}}
$$

where the pilot sequence and the channel noise have dispersion parameters $\gamma$ and $\gamma_{N_{\text {channel }}}$, respectively. Similarly, the $B E R$ is computed as the faulty number of bits divided by the total number of transmitted bits in the logarithmic scale where a total of one thousand bits have been transmitted and compared to obtain the BER vs. MSNR.

\section{Performance Evaluation}

The associated parameters of SRCS have been taken as ' $m=D=3$ ' and ' $N=500$ ' which are pre-decided between the transmitter and the intended receiver. The MSNR have been

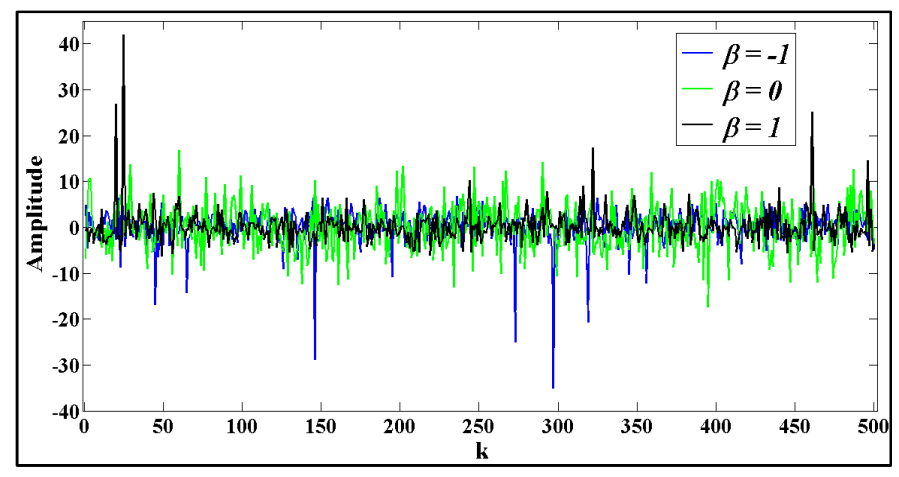

Fig. 4: Pilot Sequences taken from $\mathrm{S}_{\boldsymbol{\alpha}=\mathbf{1 . 5}}(\beta, \boldsymbol{\gamma}=1, \mu=0)$

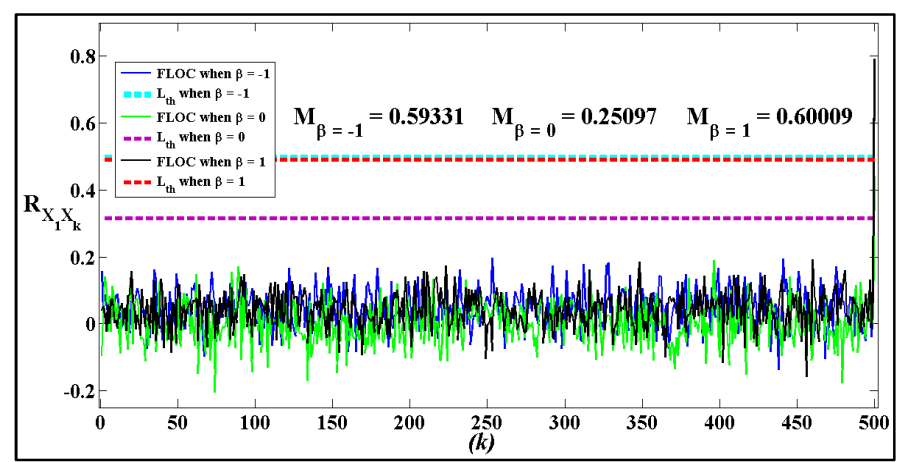

Fig. 5: FLOCs of $\mathbf{X}_{1} \mathbf{X}_{\mathbf{k}}$ for Pilot Sequences taken from $S_{\alpha=1.5}(\beta$, $\boldsymbol{\gamma}=1, \mu=0$ ) with corresponding threshold margins ' $\mathrm{M}$ ' 
taken as $-4 \mathrm{~dB}$ to acquire Figure 2, 3, 4 and 5.

\section{A. Pilot Sequence Analysis}

As shown in Fig. 2 and 4, various versions of the noisy Pilot symbol $X_{1}$, i.e. $X_{1}+G$, have been generated by varying $\alpha$ and $\beta$. It has been observed that the amplitude of $X_{1}$ increases with the decrease in $\alpha$, i.e. $X_{1}$ becomes more impulsive, and as $\beta$ approaches to zero, i.e. $X_{1}$ becomes less skewed. As a consequence shown in Fig. 3 and 5 , the difference between $\|R\|_{\infty}$ and $\left\|R_{\text {red }}\right\|_{\infty}$, increases with the decrease in $\alpha$ and as $\beta$ approaches to zero, hence, resulting in bigger $\mathbf{M}$. Similarly, the difference between $\|R\|_{\infty}$ and $\left\|R_{\text {red }}\right\|_{\infty}$, decreases with the increase in $\alpha$ and as $\beta$ approaches to its extreme value of absolute one, hence, resulting in smaller $\mathbf{M}$.

\section{B. Experimental Results}

It is predicted by the proposed optimization criterion, that the versions of the noisy pilot symbols $\boldsymbol{X}_{\mathbf{1}}$ possessing bigger $\mathbf{M}$ in Fig. 3, i.e. $\boldsymbol{X}_{\mathbf{1}} \sim \mathrm{S}_{\alpha=0.5}(\beta=1, \gamma, \mu)$ and $\boldsymbol{X}_{\mathbf{1}} \sim \mathrm{S}_{\alpha=0.5}$ $(\beta=-1, \gamma, \mu)$, and in Fig. 5, i.e. $\boldsymbol{X}_{\mathbf{1}} \sim \mathrm{S}_{\alpha=1.5}(\beta=1, \gamma, \mu)$ and $\boldsymbol{X}_{\mathbf{1}} \sim$ $\mathrm{S}_{\alpha=1.5}(\beta=-1, \gamma, \mu)$, should give better BER performance of SRCS. Therefore, it can be seen in Fig. 6 , that slanting $\beta$ of $\boldsymbol{X}_{\mathbf{1}}$ towards its extreme left, i.e. $\beta=-1$, or towards its extreme right, i.e. $\beta=1$, results in efficient BER performance of SRCSs. However, decreasing $\beta$ or slanting $\beta$ of $\boldsymbol{X}_{\mathbf{1}}$ towards its mid value, i.e. $\beta=0$, results in worst BER performance of SRCSs. Similarly, increasing the impulsiveness of $\boldsymbol{X}_{\mathbf{1}}$, i.e. decreasing $\alpha$, results in efficient BER performance of SRCSs. However, decreasing the impulsiveness of $\boldsymbol{X}_{\mathbf{1}}$, i.e. increasing $\alpha$, results in worst BER performance of SRCSs

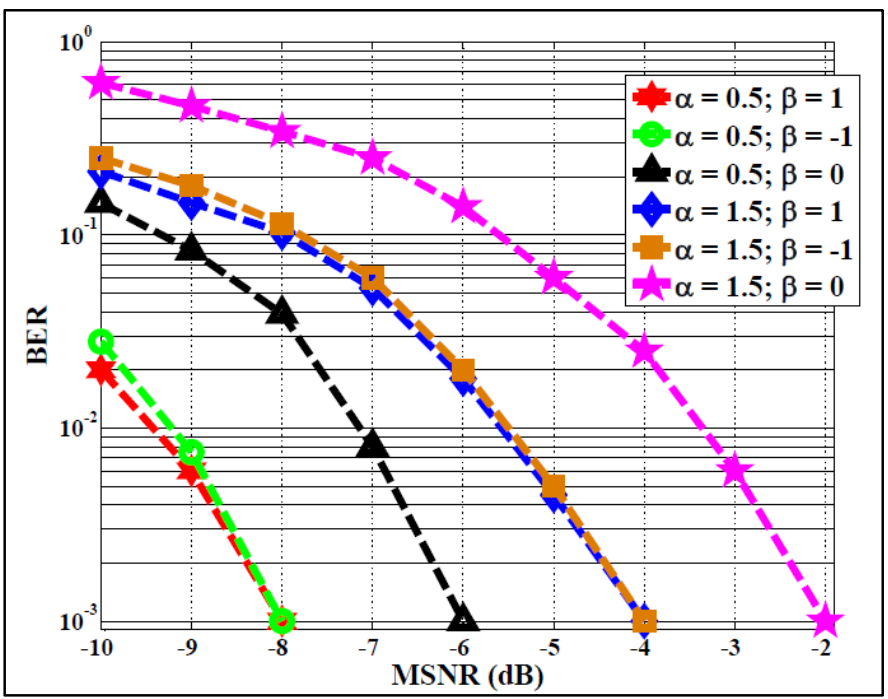

Fig. 6: BER vs. MSNR performance of S-RCS for various ' $\alpha$ ' and ' $\beta$ '

\section{CONCLUSION}

The optimization of Fractional lower order covariance based synchronization method for Random communication systems has been performed by the proposed criterion, i.e. FLOC Margin. The introduced criterion assists the transmitter and the intended receiver in pre-deciding the specific value of characteristic exponent ' $\alpha$ ' and impulsiveness parameter ' $\beta$ ' for pilot sequence generation. By choosing the parameter values of
' $\alpha$ ' and ' $\beta$ ' from the prescribed range, the detection ability of the Fractional lower order covariance based Correlators can be enhanced to the optimum level, hence, the received pilot sequence can be filtered out and recognized comfortably by the intended receiver. Therefore, precise synchronization can be established in minimum duration by utilizing the proposed criterion.

Since, Synchronized Random Communication Systems utilizes the pilot sequence which has been generated from alpha-stable distribution; therefore, the proposed criterion has been made to increase the performance of the FLOCCs. However, the concept can be applied to other threshold based communication systems as well to improve the efficiency of correlators, detectors or estimators at the receiver side which are concerns of ongoing study.

\section{REFERENCES}

[1] Basore, B.L.: 'Noise-like signals and their detection by correlation', Ph.D. Thesis, MIT, Cambridge, MA, 1952.

[2] A.B. Salberg and A. Hanssen, "Secure digital communications by means of stochastic process shift keying," in Proc. Int. Conf. Signals, Systems, and Computers, PacificGrove, USA, October 1999, pp. 1523-1527.

[3] M.E. Cek and F.A Savaci, "Stable non-Gaussian noise parameter modulation in digital communication," IET Electronics Letters, vol. 45, no. 24, pp. 1256-1257, 2009.

[4] M.E. Cek, "Covert communication using skewed $\alpha$-stable distributions," IET Electronics Letters, vol. 51, no. 1, pp. 116-118, 2015.

[5] M.E. Cek, "M-ary alpha-stable noise modulation in spread-spectrum communication," Fluc. and Noise Letters, vol. 14, no. 3, 1550022, 2015.

[6] Z.J. Xu, K. Wang, Y. Gong, W.D. Lu and J.Y. Hua, "Structure and performance analysis of an S $\alpha$ S-based digital modulation system," IET Communications, vol. 10, no. 11, pp.1329-1339, 2016.

[7] Z.J. Xu, K. Wang, Y. Gong, W.D. Lu and J.Y. Hua, "A Covert Digital Communication System Based on Joint Normal Distribution," IET Communications, 2017, vol. 11, no. 8, pp. 1282-1290

[8] A. Ahmed and F.A Savaci, "Random Communication System Based on Skewed Alpha-Stable Levy Noise Shift Keying," Fluc. and Noise Letters, vol. 16, no. 3, 1750024, 2017.

[9] A. Ahmed and F.A Savaci, "Measure of Covertness based on the imperfect synchronization of an eavesdropper in Random Communication Systems," in $10^{\text {th }}$ Int. Conf. on Elec. and Elec. Eng. (ELECO), Bursa Turkey, Nov. 2017.

[10] A. Ahmed and F.A Savaci, "Synchronization of alpha-stable levy noise based random communication systems (In-press)," IET Communications, 2017.

[11] G. Samorodnitsky and M. Taqqu, Stable non-gaussian random processes: stochastic models with infinite variance, C.\&Hall/CRC, 1994

[12] A. Janicki and A. Weron, Simulation and chaotic behavior of a-stable stochastic processes, C.\&Hall/CRC, 1994.

[13] E.E. Kuruog `lu, "Density parameter estimation of skewed alpha-stable distributions," IEEE Transaction on Signal Processing, vol. 49, no. 10, pp. 2192-2201, 2001. 